\title{
Monoclonal Antibody CD19
}

National Cancer Institute

\section{Source}

National Cancer Institute. Monoclonal Antibody CD19. NCI Thesaurus. Code C2523.

A mouse anti-human CD19 monoclonal antibody (MoAb). CD19 as well as CD22 are cell surface antigens present on a majority of B lymphocytes, including early B progenitor cells. Anti-CD19 MoAb, either alone or in combination with other immunotherapeutic agents, may be used in management of B-cell malignancies. 